\title{
An approach to duality on abelian precompact groups
}

\author{
M. J. Chasco and E. Martín-Peinador \\ (Communicated by S. A. Morris)
}

\begin{abstract}
We prove that every dense subgroup of a topological abelian group has the same 'convergence dual' as the whole group. By the 'convergence dual' we mean the character group endowed with the continuous convergence structure. We draw as a corollary that the continuous convergence structure on the character group of a precompact group is discrete and therefore a non-compact precompact group is never reflexive in the sense of convergence. We do not know if the same statement holds also for reflexivity in the sense of Pontryagin; at least in the category of metrizable abelian groups it does.
\end{abstract}

\section{Introduction and preliminaries}

The existence and properties of proper dense subgroups of topological groups has received much attention from different points of view, and there are many deep results concerning for example the class of pseudocompact groups. Some properties $\mathscr{P}$ like metrizability, admit the following statement: a dense subgroup $H$ of a topological group $G$ has $\mathscr{P}$ if and only if $G$ has $\mathscr{P}$. So there are properties for which a dense subgroup already gives the information of how the whole group behaves with respect to it. In the present paper we are mainly concerned with how dense subgroups of locally compact abelian groups behave with respect to different sorts of 'reflexivity', and how the duals of such subgroups determine the dual of the whole group.

By a continuous character on an abelian topological group $G$ we understand as usual a continuous homomorphism from $G$ into the unit circle $\mathbb{T}$ of the complex plane, endowed with the Euclidean topology. Under multiplication the continuous characters on $G$ constitute a group denoted by $\Gamma G$, and called the dual group of $G$. A continuous character defined on a dense subgroup $H$ of a topological abelian group $G$ can be extended to the whole group, due to the fact that characters are uniformly continuous mappings. This leads to the algebraic identification of the corresponding dual groups $\Gamma G$ and $\Gamma H$.

On the other hand, inspired by the Pontryagin-van Kampen duality theory, it is natural to consider the compact open topology for the dual of a group. In general

This work was partially supported by MTM2006-03036 and FEDER Funds 
we will write $G^{\wedge}$ for $\Gamma G$ endowed with the compact open topology, and call it the Pontryagin dual or simply the dual group. If again $H$ is a dense subgroup of an abelian topological group $G, H^{\wedge}$ may be quite different from $G^{\wedge}$. The above mentioned algebraic identification does not extend to the topological context.

This fact gives rise to a new class of groups. A topological abelian group $G$ will be called a determined group if for any dense subgroup $H<G$, the restriction mapping from $G^{\wedge}$ to $H^{\wedge}$ is a topological isomorphism; roughly speaking, if the respective dual groups equipped with the compact open topology coincide algebraically and topologically. Metrizable abelian groups are determined, as proved by the first author in [9], and independently in [1]. The name 'determined group' appeared for the first time in Raczkowski's doctoral thesis [15], where she proves that even compact groups may be non-determined.

Throughout the paper all groups considered will be abelian. A basis of neighborhoods of the neutral element for the compact open topology $\tau_{c o}$ is given by the sets $\left(K, \mathbb{T}_{\varepsilon}\right):=\left\{\varphi \in \Gamma G: \varphi(K) \subseteq \mathbb{T}_{\varepsilon}\right\}$, where $\mathbb{T}_{\varepsilon}=\{z \in \mathbb{T}:|z-1| \leqslant \varepsilon\}$ and $K$ is a compact subset of $G$. Clearly $G^{\wedge}$ is a Hausdorff topological abelian group. The polar of a subset $A \subset G$ is the set

$$
A^{\triangleright}:=\left\{\psi \in \Gamma G: \psi(A) \subseteq \mathbb{T}_{+}\right\}
$$

where $\mathbb{T}_{+}=\mathbb{T}_{\sqrt{2}}$. The inverse polar of a subset $B$ of $\Gamma G$ is the set

$$
B^{\triangleleft}:=\left\{x \in G: \psi(x) \in \mathbb{T}_{+}, \forall \psi \in B\right\} .
$$

The quasi-convex hull of a subset $A \subset G$ is qc $(A):=A^{\triangleright \triangleleft}$; the set $A$ is said to be quasiconvex if $\operatorname{qc}(A)=A$. A topological abelian group is locally quasi-convex if it admits a basis of neighborhoods of zero consisting of quasi-convex sets. A natural example of a locally quasi-convex group is the underlying group of a locally convex space; see [2].

A topological abelian group $G$ is said to be reflexive if the canonical mapping $\alpha_{G}: G \rightarrow G^{\wedge \wedge}$ given by $\alpha_{G}(x)(\kappa)=\kappa(x)$ is a topological isomorphism. The class of reflexive groups is a natural extension of that of locally compact abelian groups (LCA-groups) via the well-known theorem of Pontryagin and van Kampen, which states that LCA-groups are reflexive. Furthermore, the dual group $G^{\wedge}$ of a locally compact abelian group $G$, is again locally compact and the evaluation mapping $e_{G}: G \times G^{\wedge} \rightarrow \mathbb{T}$ (defined by $\left.e_{G}(g, \xi)=\xi(g)\right)$ is continuous.

Continuity here seldom arises outside the class of LCA-groups; for an arbitrary group $G$, if the continuity of the evaluation mapping is to be achieved, some $\tau_{c o^{-}}$ convergent nets of $\Gamma G$ must be killed. In doing so, a new convergence structure is obtained which is no longer a topology; it is called the continuous convergence structure. If a group $G$ is endowed with a convergence structure which is compatible with the algebraic structure of $G$, then it is said to be a convergence group. All topological groups can be treated as convergence groups. See [4] for a more detailed formulation.

If $G$ is a convergence abelian group, then the continuous convergence structure $\Lambda$ on $\Gamma G$ is defined as the coarsest among all those convergence structures in $\Gamma G$ 
which make continuous the evaluation mapping $e_{G}: G \times \Gamma G \rightarrow \mathbb{T}$. If $G$ is locally compact then the continuous convergence structure on $\Gamma G$ coincides with the compact-open topology; in other words they have the same convergent nets. It can be explicitly defined either by its convergent filters or by its convergent nets, as we do next.

A filter $\mathscr{F}$ in $\Gamma G$ converges in $\Lambda$ to an element $\xi \in \Gamma G$ if for every $x \in G$ and every filter $\mathscr{H}$ in $G$ that converges to $x, \omega(\mathscr{F} \times H)$ converges to $\xi(x)$ in $\mathbb{T}$ (here, $\mathscr{F} \times \mathscr{H}$ denotes the filter generated by the products $F \times H$, where $F \in \mathscr{F}, H \in \mathscr{H}$ and $\omega(\mathscr{F} \times H)$ is the filter generated by $\omega(F \times H):=\{f(x) ; f \in F, x \in H\})$. Equivalently, a net $\left\{\xi_{\alpha}, \alpha \in \mathscr{A}\right\}$ in $\Gamma G$ is $\Lambda$-convergent to $\xi \in \Gamma G$ (or continuously convergent) if for every net $\left\{x_{\beta}, \beta \in \mathscr{B}\right\}$ in $G$ such that $x_{\beta} \rightarrow x$, the net $\left\{\xi_{\alpha}\left(x_{\beta}\right)\right\}$, indexed by the product $\mathscr{A} \times B$ and directed in the natural way, converges in $\mathbb{T}$ to $\xi(x)$. Every convergent filter in $\Lambda$ has a compact element, that is to say, $\Lambda$ is a locally compact structure, see [5].

Both pointwise multiplication in $\Gamma G$ and taking the inverse are continuous operations with respect to the continuous convergence structure. Therefore it makes sense to call the pair $(\Gamma G, \Lambda)$ the convergence dual or the BB-dual of $G$, henceforth denoted by $\Gamma_{c} G$. The corresponding duality has been systematically studied in [4]. A convergence group $G$ is called BB-reflexive if it is canonically isomorphic to its convergence bidual $\Gamma_{c} \Gamma_{c} G$. We introduced this terminology in [8], after Binz and Butzmann, who were the first to apply successfully the theory of duality to convergence vector spaces and convergence abelian groups.

The topology on $G$ of pointwise convergence in the elements of $\Gamma G$, denoted by $\sigma(G, \Gamma G)$, is usually called the Bohr topology of $G$; the symbol $G_{b}$ stands for the group $G$ endowed with its Bohr topology. The completion of $G_{b}$ is usually called the Bohr compactification of $G$. It is denoted by $b G$ and it is exactly $\left(G_{d}^{\wedge}\right)^{\wedge}$, where $G_{d}^{\wedge}$ denotes the character group $\Gamma G$ endowed with the discrete topology.

The sets $\Gamma G_{b}$ and $\Gamma G$ coincide (see [11]), although in general the compact-open topology in $\Gamma G_{b}$ is finer than the compact-open topology in $\Gamma G$. Nevertheless $\left(\Gamma G_{b}, \tau_{c o}\right)$ and $\left(\Gamma G, \tau_{c o}\right)$ coincide algebraically and topologically for a nuclear group $G$ (see [3]). The class of nuclear groups is a large class of topological groups which includes LCA groups, and is closed by taking subgroups, arbitrary products and quotients (see [2]). Similarly, we denote by $\sigma(\Gamma G, G)$ the topology in $\Gamma G$ of pointwise convergence on the elements of $G$.

\section{The convergence-dual of a dense subgroup}

The main result of this section is the fact that all abelian groups are determined from the point of view of convergence, as expressed in Theorem 3. This leads to the simple observation that the convergence dual of a group endowed with its Bohr topology is discrete (Corollary 6).

Lemma 1. Let $H$ be a dense subgroup of a topological abelian group $G$. Then $\Gamma H$ may be identified with $\Gamma G$, and the corresponding families of equicontinuous subsets coincide, i.e. $\mathscr{E}(\Gamma H)=\mathscr{E}(\Gamma G)$. 
Proof. The first assertion is a consequence of the well-known fact that every continuous character on a dense subgroup can be continuously extended to a character on the whole group in a unique way. From now on we identify the sets $\Gamma G$ and $\Gamma H$. For the second assertion, the inclusion $\mathscr{E}(\Gamma H) \supset \mathscr{E}(\Gamma G)$ follows directly from the definition of equicontinuity. Conversely, let $M \in \mathscr{E}(\Gamma H)$, and let $V$ be an open neighborhood of the neutral element of $H$ such that $M \subset V^{\triangleright}$. Denote by $W$ an open neighborhood of the neutral element of $G$, with $W \cap H=V$. We have

$$
V^{\triangleright}=(W \cap H)^{\triangleright}=(\overline{W \cap H})^{\triangleright}=\bar{W}^{\triangleright} .
$$

Thus $M \subset \bar{W}^{\triangleright}$ and so $M \in \mathscr{E}(\Gamma G)$.

Lemma 2. A net $\left\{\xi_{\alpha}, \alpha \in \mathscr{A}\right\}$ in $\Gamma G$ is $\Lambda$-convergent to an element $\xi \in \Gamma G$ if and only if its range is eventually equicontinuous and it converges to $\xi$ in the pointwise topology of $\Gamma G$.

Proof. The proof is a direct computation. By eventually equicontinuous range we mean that there exists $\alpha_{0} \in \mathscr{A}$ such that $\left\{\xi_{\alpha}, \alpha \geqslant \alpha_{0}\right\} \cup\{\xi\}$ is an equicontinuous subset.

Theorem 3. Let $H$ be a dense subgroup of a topological abelian group $G$. The continuous convergence structures in $\Gamma G$ and in $\Gamma H$ coincide.

Proof. Let $\Lambda(\Gamma G)$ and $\Lambda(\Gamma H)$ denote the continuous convergence structures in $\Gamma G$ and in $\Gamma H$ respectively. This distinction is convenient because $\Gamma G$ and $\Gamma H$ are so far identified as sets, but not as convergence groups. Let $S=\left\{\xi_{\alpha}, \alpha \in \mathscr{A}\right\}$ be a net in $\Gamma G=\Gamma H$. By the definition of the continuous convergence structure, if $S$ converges to $\xi$ in $\Lambda(\Gamma G)$ it obviously converges to $\xi$ in $\Lambda(\Gamma H)$.

For the converse implication suppose now that $S \rightarrow \xi$ in $\Lambda(\Gamma H)$. Its range is eventually equicontinuous in $\Gamma H$ (without loss of generality suppose that $S$ has equicontinuous range) and by Lemma 1 it is also equicontinuous in $\Gamma G$. Therefore, a zero-neighborhood $U \subset G$ can be found such that $S=\left\{\xi_{\alpha}, \alpha \in \mathscr{A}\right\} \subset U^{\triangleright}$. Since $U^{\triangleright} \subset \mathbb{T}^{G}$ is clearly compact in the pointwise convergence topology $\sigma(\Gamma G, G)$, the net $S$ has a $\sigma(\Gamma G, G)$-convergent subnet $T$, say $T \rightarrow \psi \in \Gamma G$.

On the other hand, a $\sigma(\Gamma G, G)$-convergent net with equicontinuous range is also $\Lambda(\Gamma G)$-convergent with the same limit. Therefore the subnet $T$ must converge to $\xi$. The same argument proves that every subnet of $\left\{\xi_{\alpha}, \alpha \in \mathscr{A}\right\}$ has in turn a subnet converging to $\xi$ in $\Lambda(\Gamma G)$. Thus $\xi_{\alpha} \rightarrow \xi$ in $\Lambda(\Gamma G)$.

In [4] it is proved that a topological group and its completion admit the same convergence dual. This is a consequence of the above theorem.

Corollary 4. A proper dense subgroup $H$ of a LCA-group $G$ cannot be BB-reflexive. If $H$ is metrizable then $H$ cannot be Pontryagin reflexive. 
Proof. By Theorem 3, the group $\Gamma_{c} H$ is bicontinuously isomorphic to $\Gamma_{c} G$. Since $G$ is a LCA-group, $\Gamma_{c} G$ coincides with $G^{\wedge}$ which is also locally compact. For the same reason, $\Gamma_{c} \Gamma_{c} G$ coincides with $G^{\wedge \wedge}$. Now $\Gamma_{c} \Gamma_{c} H$ is bicontinuously isomorphic to $\Gamma_{c} \Gamma_{c} G$, which is a LCA-group. Therefore $\Gamma_{c} \Gamma_{c} H$ cannot be bicontinuously isomorphic to $H$, for otherwise $H$ would be locally compact, and this contradicts the density of $H$ in $G$.

The second assertion comes from the fact that Pontryagin reflexivity and BBreflexivity are equivalent for metrizable groups (see [9]).

Next we draw some consequences for the Bohr topology on a topological group.

Corollary 5. For an abelian non-compact topological group $G, G_{b}$ cannot be BBreflexive.

Corollary 6. For an abelian topological group $G, \Gamma_{c} G_{b}$ is discrete.

Proof. The group $G_{b}$ is a dense subgroup of $b G$, and by Theorem $3, \Gamma_{c} G_{b}$ is bicontinuously isomorphic to $\Gamma_{c}(b G)$. Since $b G$ is compact, $\Gamma_{c}(b G)$ is discrete.

Remark 1. Observe that from Corollary 6, we can claim that the Bohr compactification $b G$ of a topological group $G$ is exactly the convergence bidual $\Gamma_{c} \Gamma_{c} G_{b}$. This simple observation seems not to have been stated before in the literature.

\section{The Pontryagin dual of a dense subgroup}

In this section we deal mainly with LCA-groups. The continuous convergence structure $\Lambda$ in the dual of a LCA-group $G$ coincides with the convergence defined by the compact-open topology $\tau_{c o}$. Nevertheless this assertion does not hold for dense subgroups of $G$. Next, we study some requirements that a dense subgroup of a LCAgroup must satisfy in order that its character group with the compact-open topology coincides with that of the whole group.

Proposition 7. Let $H$ be a dense subgroup of a LCA-group G. The following assertions are equivalent:

(a) the dual groups $H^{\wedge}$ and $G^{\wedge}$ are topologically isomorphic, that is, $\tau_{c o}(\Gamma H)=\tau_{c o}(\Gamma G)$

(b) the continuous convergence $\Lambda(\Gamma H)$ coincides with the convergence defined by $\tau_{c o}(\Gamma H)$;

(c) the evaluation mapping $e_{H}: H \times H^{\wedge} \rightarrow \mathbb{T}$ is continuous;

(d) the dual group $H^{\wedge}$ is locally compact and $\alpha_{H}: H \rightarrow H^{\wedge \wedge}$ is continuous. 
Proof. (a) $\Rightarrow$ (b) Since $G$ is locally compact, $\Lambda(\Gamma G)=\tau_{c o}(\Gamma G)$. This together with the equality $\Lambda(\Gamma G)=\Lambda(\Gamma H)$ obtained in Theorem 3 and (a) gives $\Lambda(\Gamma H)=\tau_{c o}(\Gamma H)$.

(b) $\Rightarrow$ (c) For the LCA group $G$ the evaluation mapping $e_{G}: G \times G^{\wedge} \rightarrow \mathbb{T}$ is continuous and $\Lambda(\Gamma G)=\tau_{c o}(\Gamma G)$. By Theorem 3 and $(\mathrm{b}), e_{H}: H \times H^{\wedge} \rightarrow \mathbb{T}$ is the restriction of $e_{G}$, and therefore it is continuous.

(c) $\Rightarrow$ (d) This appears in [14].

(d) $\Rightarrow$ (a) Since $\alpha_{H}$ is continuous, any $\tau_{c o}$-compact subset $C \subset \Gamma H$ is equicontinuous in $\Gamma H$ and by Lemma 1 also in $\Gamma G$. This implies that $C$ is $\tau_{c o}$-compact in $\Gamma G$. Taking into account that $H^{\wedge}$ is locally compact, $\tau_{c o}(\Gamma H)$ and $\tau_{c o}(\Gamma G)$ are two k-topologies with the same compact subsets, and thus they must coincide.

Remark 2. The continuity of the evaluation mapping $e_{H}: H \times H^{\wedge} \rightarrow \mathbb{T}$ is a strong requirement. In fact, in the class of reflexive groups (see [13]) the continuity of $e_{H}$ implies that the group $H$ must be locally compact. The same assertion is obtained for the class of topological groups with the property that the quasi-convex hull of compact sets are again compact (see [14]).

The topological modification of $\Lambda$, denoted by $\tau_{\Lambda}$, is the topology on $\Gamma G$ defined as follows: a subset $C$ of $\Gamma G$ is closed in $\tau_{\Lambda}$ if and only if it contains the limit points of every $\Lambda$-convergent net contained in $C$. It is straightforward to check that $\tau_{\Lambda}$ is in fact a topology, but in general it fails to be a group topology. By its definition $\tau_{\Lambda}$ is the finest topology among those coarser than $\Lambda$. It is finer than the compact-open topology and if $G$ is metrizable, then $\tau_{\Lambda}=\tau_{c o}$; see [9].

For further use we mention the following facts which can be easily deduced from our previous considerations:

Lemma 8. Let $G$ be a topological group and $L \subset \Gamma G$. Then the following assertions hold.

(i) $L$ is $\Lambda$-compact if and only if it is equicontinuous and $\Lambda$-closed.

(ii) If $L$ is equicontinuous then the induced convergence $\left.\Lambda\right|_{L}$ is topological; it coincides with the convergence associated to the topology $\sigma\left(G^{\wedge}, G\right)$.

(iii) The topological modification $\tau_{\Lambda}$ of the continuous convergence in the dual of a topological abelian group $G$ is a $k$-topology.

It is proved in [7] that $\tau_{\Lambda}$ is the weak topology with respect to the family of all equicontinuous subsets of $\Gamma G$, and therefore it is the finest topology of all those which induce $\sigma(\Gamma G, G)$ in the equicontinuous subsets of $\Gamma G$. In [7] the following question is asked: if $\tau_{\Lambda}$ is a group topology in the dual of a topological group, must it be the k-extension of the corresponding compact-open topology? We provide a negative answer in the corollary below.

Proposition 9. Let $G$ be a topological group such that $\alpha_{G}: G \rightarrow G^{\wedge \wedge}$ is continuous. The topology $\tau_{\Lambda}$ is the k-extension of the compact-open topology on $\Gamma G$. 
Proof. The relationship among convergences is as follows: $\Lambda$-convergence $\Rightarrow \tau_{\Lambda^{-}}$ convergence $\Rightarrow \tau_{c o}$-convergence. If $\alpha_{G}$ is continuous every subset which is compact in the compact-open topology is also $\Lambda$-compact. Thus, the corresponding families of compact subsets coincide. By (iii) in the previous Lemma, $\tau_{\Lambda}$ is the k-extension of $\tau_{c o}$.

Corollary 10. If $G$ is a locally compact non-compact topological group then the topology $\tau_{\Lambda}$ in $\Gamma G_{b}$ is a group topology, but it is not the k-extension of the compact-open topology in the same space.

Proof. The topology $\tau_{\Lambda}$ is discrete, since the continuous convergence structure in $\Gamma G_{b}$ is already discrete (see Corollary 6). On the other hand, since Bohr-compact subsets coincide with the original compact subsets of $G$ (by Glicksberg's theorem), the compact-open topologies for the groups $\Gamma G$ and $\Gamma G_{b}$ are identical. Therefore $\left(G_{b}\right)^{\wedge}=G^{\wedge}$ is a k-space which cannot be discrete, for otherwise $\left(G_{b}\right)^{\wedge \wedge}=G^{\wedge \wedge}$ would be compact, and by Pontryagin's Theorem also $G$ would be compact.

If the dual of a precompact non-compact group is discrete, then the group itself cannot be reflexive. From Corollary 10 it is clear that there exist also non-reflexive precompact groups with non-discrete duals. This leads us to the following:

Question. Is a precompact abelian group necessarily non-reflexive?

Remark 3. From Corollary 10 and Proposition 9 one can conclude that for a noncompact LCA-group $G$, the canonical mapping $\alpha_{G_{b}}$ is not continuous.

\section{Topological abelian groups whose compact subsets are finite}

Lemma 11. If the compact subsets of a topological group $G$ are finite, then $G^{\wedge}$ is topologically isomorphic to $\left(G^{\wedge}\right)_{b}$.

Proof. Since compact subsets of $G$ are finite, the compact open topology on $\Gamma G$ coincides with $\sigma(\Gamma G, G)$. On the other hand $G^{\wedge \wedge}=(\Gamma G, \sigma(\Gamma G, G))^{\wedge}$ can be algebraically identified with $G$, since its elements are the evaluation mappings on points of $G$. Now the Bohr topology in $G^{\wedge}$ is precisely $\sigma(\Gamma G, G)$.

Next we collect some results about $P$-groups. We recall that a topological space $X$ is a $P$-space if all of its $G_{\delta}$-sets are open. An abelian topological group which is a $P$-space is called a $P$-group.

Lemma 12. Suppose that $G$ is a P-group. Then the following assertions hold:

(1) compact subsets of $G$ are finite;

(2) G has a basis of neighbourhoods of the neutral element consisting of open subgroups; 
(3) G can be embedded in a product of discrete groups;

(4) $G$ is a nuclear group.

If furthermore $G$ is complete, then:

(5) $G$ is an inverse limit of discrete groups;

(6) $\alpha_{G}$ is an open isomorphism;

(7) $G$ is BB-reflexive;

(8) $G^{\wedge}$ is precompact and $\alpha_{G^{\wedge}}$ is continuous.

Proof. (1) and (2) are well-known facts and (3) can be obtained as a corollary of (2). The fact that $G$ is nuclear can be derived from [2, (7.6), (7.3) and (7.5)]. For (5) see $[12,(3.5)]$. Assertion (6) is $[1,(29.5)]$ and (7) can be found in $[6,(4.4)]$.

For (8), we take into account that as a consequence of (1), the compact-open topology in $\Gamma G$ coincides with that of pointwise convergence $\sigma(\Gamma G, G)$, and therefore $G^{\wedge}$ can be identified with a subgroup of a product of copies of $\mathbb{T}$. On the other hand, if $\alpha_{G}$ is open and bijective then the compact subsets of $G^{\wedge \wedge}$ are finite, therefore equicontinuous, and this in turn implies that $\alpha_{G^{\wedge}}$ is continuous; see [1, (5.10)].

Let us give an example of a $P$-group and its dual group.

Example. Consider the cyclic group $\mathbb{Z}(n)$ of order $n \geqslant 2$ and a set $I$ of cardinality greater or equal than continuum and take the product $\mathbb{Z}(n)^{I}$ of copies of $\mathbb{Z}(n)$ indexed by $I$. Let $G$ be the direct sum $\mathbb{Z}(n)^{(I)}$, with the group topology whose basis of zero-neighborhoods consists of the direct sums $\bigoplus_{i \in I} U_{i}$, where $U_{i}=\mathbb{Z}(n)$ except for a countable set $H \subset I$, and $U_{i}=\{0\}$ for all $i \in H$.

It is clear that $G$ is a $P$-group and easy computations allow us to see that its Pontryagin dual $G^{\wedge}$ is the $\Sigma$-product $\sum \mathbb{Z}(n)$, as a topological subgroup of $\mathbb{Z}(n)^{I}$ with the product topology.

By Lemma 11 the group $G^{\wedge}$ is precompact. On the other hand, the dual of the $\Sigma$ product $\sum \mathbb{Z}(n)$ coincides with the dual of the full product $\mathbb{Z}(n)^{I}$ since it is proved in [10] that the direct sum of compact groups determines the corresponding product. Then the Pontryagin bidual $G^{\wedge \wedge}$ is $\mathbb{Z}(n)^{(I)}$ with the discrete topology.

Remark 4. Observe that $\alpha_{G^{\wedge}}$ in the above example is continuous, in contrast to Remark 3.

We do not know if the bidual of any $P$-group is necessarily discrete. This is relevant to the following question of S. Hernández: is every Pontryagin reflexive $P$-group discrete? A negative answer would solve the question mentioned after Corollary 10. In fact, if there exists a non-discrete Pontryagin reflexive $P$-group, then its dual must be precompact and reflexive. 


\section{References}

[1] L. Außenhofer. Contributions to the duality theory of abelian topological groups and to the theory of nuclear groups. Dissertationes Math. (Warszawa 1999).

[2] W. Banaszczyk. Additive subgroups of topological vector spaces. Lecture Notes in Math. 1466 (Springer-Verlag, 1991).

[3] W. Banaszczyk and E. Martín-Peinador. The Glicksberg theorem on weakly compact sets for nuclear groups. Ann. New York Acad. Sci. 788 (1996), 34-39.

[4] R. Beattie and H. P. Butzmann. Convergence structures and applications to functional analysis (Kluwer Academic, 2002).

[5] M. Bruguera and M. J. Chasco. Strong reflexivity of abelian groups. Czech. Math. J. 51 (2001), 213-224.

[6] M. Bruguera, M. J. Chasco, E. Martín-Peinador and V. Tarieladze. Completeness properties of locally quasi-convex groups. Topology Appl. 111 (2001), 81-93.

[7] M. Bruguera and E. Martín-Peinador. Banach-Dieudonné theorem revisited. J. Austral. Math. Soc. 75 (2003), 1-15.

[8] M. J. Chasco and E. Martín-Peinador. Binz-Butzmann duality versus Pontryagin duality. Arch. Math. (Basel) 63 (1994), 264-270.

[9] M. J. Chasco. Pontryagin duality for metrizable groups. Arch. Math. (Basel) 70 (1998), 22-28.

[10] W. W. Comfort, S. Raczkowski and F. J. Trigos-Arrieta. The dual group of a dense subgroup. Czech. Math. J. 54 (2004), 509-533.

[11] W. W. Comfort and K. A. Ross. Topologies induced by groups of characters. Fund. Math. 55 (1964), 283-291.

[12] G. Itzkowitz. Projective limits and balanced topological groups. Topology Appl. 110 (2001), 163-183.

[13] E. Martín-Peinador. A reflexive admissible topological group must be locally compact. Proc. Amer. Math. Soc. 123 (1995), 3563-3566.

[14] E. Martín-Peinador and V. Tarieladze. A property of Dunford-Pettis type in topological groups. Proc. Amer. Math. Soc. 132 (2003), 1827-1837.

[15] S. U. Raczkowski. Totally bounded topological group topologies. Ph.D. thesis. Wesleyan University (1999).

Received 16 April, 2007; revised 5 September, 2007

M. J. Chasco, Dept. de Física y Matemática Aplicada, Universidad de Navarra, Spain E-mail:mjchasco@unav.es

E. Martín-Peinador, Dept. de Geometría y Topología, Universidad Complutense de Madrid, Spain

E-mail: peinador@mat.ucm.es 